\title{
Estudio microbiológico de la periimplantitis: Presentación de 9 casos clínicos
}

\author{
QUINTEROS BORGARELLO, M.* \\ DELGADO MOLINA, E.** \\ SÁNCHEZ GARCÉS, M $\mathbf{M}^{\mathrm{a}}$ A. *** \\ BERINI AYTÉS, L.**** \\ GAY ESCODA, C. *****
}

\author{
Quinteros Borgarello, Milva; Delgado Molina, Esther; Sánchez \\ Garcés, M. ${ }^{a}$ Ángeles; Berini Aytés, Leonardo; Gay Escoda, Cosme. \\ Estudio microbiológico de la periimplantitis: Presentación de 9 \\ casos clínicos. Av Periodon Implantol. 2000; 12, 137-150
}

\section{RESUMEN}

Objetivos: La periimplantitis es un proceso inflamatorio de los tejidos que rodean a un implante sometido a carga que produce la pérdida de soporte óseo. En este trabajo se muestran los resultados del estudio microbiológico y del antibiograma realizado a partir del exudado y/o del tejido periimplantario de 16 implantes funcionales con periimplantitis de 9 pacientes que fueron tratados en nuestro servicio.

Material y método: Se estudiaron 16 periimplantitis observadas en 9 pacientes; de 14 de ellas se obtuvieron muestras con puntas de papel estériles que se introdujeron en la bolsa periimplantaria. En 2 implantes las muestras procedían de tejido de granulación periimplantario. Inmediatamente después de ser obtenidas, se colocaban en placas de agar-chocolate con brain heart infusion para proceder a su cultivo.

Resultados: Los especímenes bacterianos aislados fueron: Stomatococcus, Prevotella oralis, Peptostreptococcus y Fusobacterium nucleatum. En 9 muestras fue imposible aislar una bacteria predominante debido a la complejidad de la flora. Respecto al resto de muestras, la bacteria predominante fue Stomatococcus en 3, Prevotella oralis en 1, Peptostreptococcus en 1 y Fusobacterium nucleatum en 2. Los antibiogramas mostraron una mayor sensibilidad a la asociación de la amoxicilina con el ácido clavulánico, comparada con la amoxicilina, el metronidazol o una combinación de estos dos últimos antimicrobianos.

Conclusiones: En este trabajo las bacterias asociadas más frecuentemente a la periimplantitis fueron: Stomatococcus, Prevotella oralis, Peptostreptococcus y Fusobacterium nucleatum. En ningún caso se aislaron cepas de Actinobacillus actinomycetemcomitans. Los posibles factores de riesgo asociados a periimplantitis en nuestra serie fueron los implantes recubiertos con hidroxiapatita, implantes de $3,25 \mathrm{~mm}$ de diámetro y la localización más distal del implante en las prótesis que rehabilitaban extremos libres edéntulos superiores.

\section{PALABRAS CLAVE}

Implantes dentales; microbiología de la periimplantitis.

**** Profesor Titular de Patología Quirúrgica Bucal y Maxilofacial. Profesor del Máster de Cirugía e Implantología Bucal. Facultad de Odontología de la Universidad de Barcelona

***** Catedrático de Patología Quirúrgica Bucal y Maxilofacial. Director del Máster de Cirugía e Implantología Bucal. Facultad de Odontología de la Universidad de Barcelona. Cirujano Maxilofacial del Centro Médico Teknon. Barcelona 


\section{INTRODUCCIÓN}

Los implantes dentales oseointegrados, utilizados cada vez más en nuestro medio, permiten ofrecer a nuestros pacientes unas prótesis confortables y funcionales, mejorando así su calidad de vida ${ }^{1}$.

La periimplantitis se define como un proceso inflamatorio que afecta a los tejidos que rodean un implante oseointegrado en función y que produce una pérdida del soporte óseo ${ }^{2}$. Si los cambios inflamatorios se observan en los tejidos blandos cercanos a un implante sometido a carga, hablamos de una mucositis periimplantaria.

Existen múltiples factores que intervienen en la etiología de la periimplantitis, aunque hay dos de más destacados: la sobrecarga biomecánica y la infección periimplantaria ${ }^{3-10}$.

Los signos y síntomas más importantes de la perimplantitis ${ }^{3}$ son:

1-Evidencia radiológica de destrucción ósea periimplantaria manteniéndose, generalmente, una zona apical sin alteraciones. En muchos casos la pérdida ósea, de aspecto radiotransparente, se produce sin que existan signos directos de movilidad del implante.

2- Destrucción ósea vertical asociada a la formación de una bolsa periimplantaria.

3- Sangrado al sondaje e incluso supuración de los tejidos periimplantarios.

4- Inflamación eventual de los tejidos periimplantarios.

5- Dolor, aunque no es un síntoma característico.

Una vez instaurada la periimplantitis, la superficie del implante queda expuesta al medio bucal y es colonizada por bacterias. El objetivo del tratamiento será recuperar la integración del implante con el hueso, es decir, una "reoseointegración". Este tratamiento se puede realizar siempre y cuando veamos que la pérdida ósea no sea superior a $2 / 3$ de su longitud o bien no exista movilidad ${ }^{11}$.

La adherencia de los fibroblastos del hueso al implante está obstaculizada por las endotoxinas, por lo que es necesario descontaminar la superficie del implante para obtener la máxima reoseointegración con las técnicas pertinentes a cada caso ${ }^{10,11}$.

El objetivo de este trabajo es mostrar, valorar y discutir los resultados del estudio microbiológico del exudado o del tejido periimplantario de 16 implantes en 9 pacientes tratados de periimplantitis en nuestro servicio.

Las muestras fueron extraídas a partir del exudado o
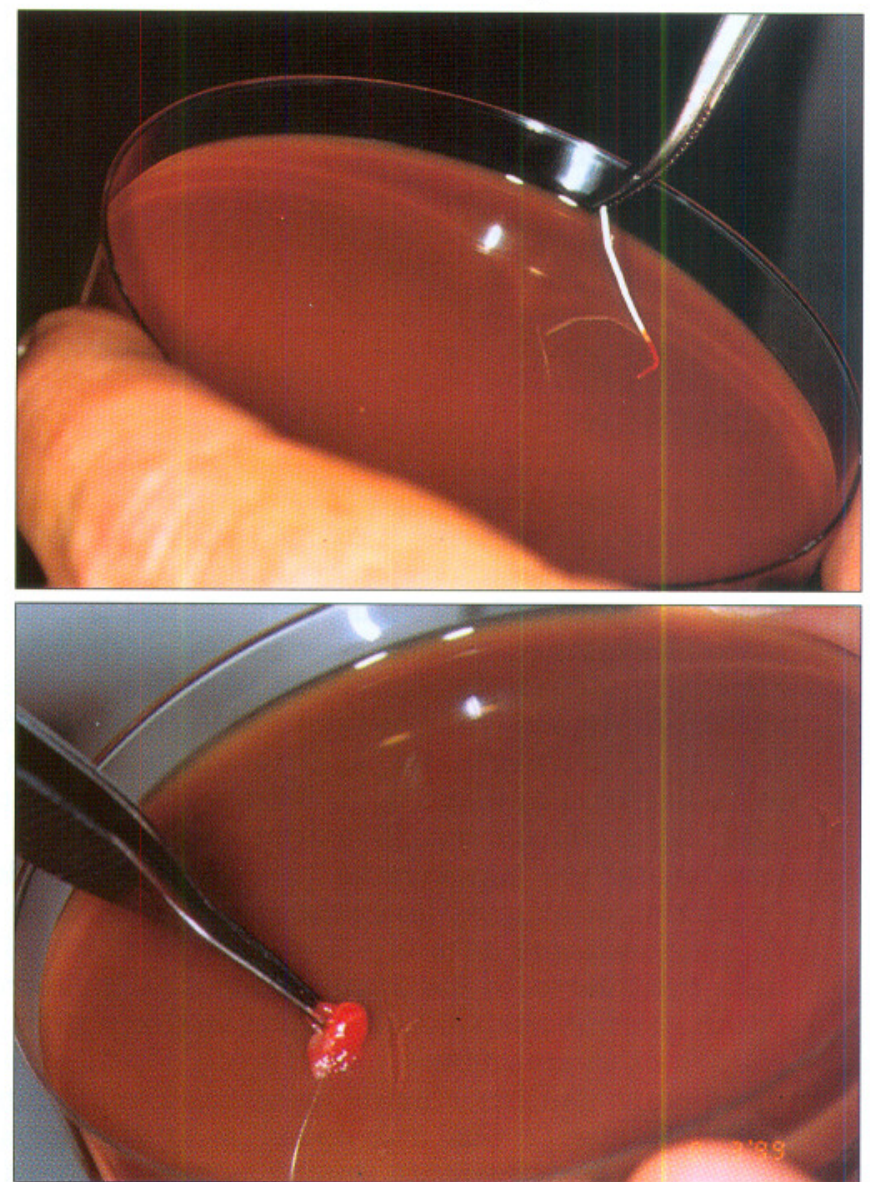

Fig. 1A.: Colocación de la punta de papel empapada en el periimplantario en el cultivo de agar-chocolate enriquecido con brain heart infusion. B.: Frotis en el cultivo de agar-chocolate del tejido de granulación adyacente a un implante con periimplantitis.

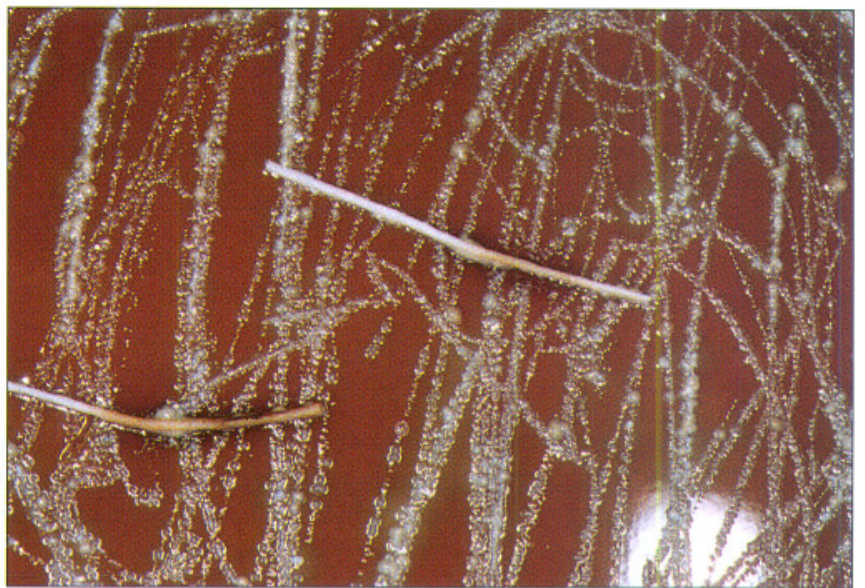

Fig. 2.: Cultivo microbiológico de dos puntas de papel en una placa de agar-chocolate enriquecido con brain heart infusion. 
del tejido periimplantario de implantes con periimplantitis. Todos estos implantes ya habían sido cargados con sus respectivas prótesis.

De los 16 implantes estudiados, en 14 se obtuvieron muestras de exudado con puntas de papel estériles que se introdujeron en la bolsa periimplantaria. En 2 implantes las muestras fueron de tejido de granulación periimplantario. A continuación, las muestras se colocaron en placas de agar-chocolate con brain heart infusion (figura 1). Las placas de Petri se introdujeron en un frasco de vidrio diseñado especialmente para su transporte en un medio anaerobio. Se realizaron cultivos de anaerobios y de aerobios para determinar las bacterias predominantes en cada muestra (figura 2), practicándose también un antibiograma para establecer el tratamiento antibiótico de elección en cada paciente. Los antimicrobianos estudiados fueron: la amoxicilina, el metronidazol y la combinación de amoxicilina con ácido clavulánico o con metronidazol. Una vez tomada la muestra, se procedía al tratamiento del implante infectado con la descontaminación de la superficie del implante, o bien con la extracción del implante afectado si la pérdida ósea era muy importante.

\section{CAsos CLÍNICOS.}

\section{CASO 1}

Varón de 39 años de edad con antecedentes de una gastrectomía subtotal realizada hace 6 años debido a un linfoma gástrico y tratamiento posterior con quimioterapia durante 6 meses. No tenía otra patología sistémica de interés. Fumador de 20 cigarrillos al día.

El paciente presentaba edentulismo parcial superior y era portador de una prótesis fija desde el 1.4. al 2.4. cuyos pilares estaban endodonciados e infraobturados. También padecía una enfermedad periodontal avanzada que nunca había sido tratada. El edentulismo en la zona mandibular correspondía a los molares del tercer y cuarto cuadrante.

En el plan de tratamiento se decidió efectuar las extracciones de los dientes superiores remanentes y colocar una prótesis completa superior inmediata. Tres meses después, y bajo sedación con óxido nitroso, se colocaron 6 implantes Calcitek Integral ${ }^{\circledR}$ de titanio recubiertos de hidroxiapatita en la posición correspondiente al 1.5., 1.3, 1.1., mesial del 2.2., 2.3. y zona del 2.4. Todas las fijaciones eran de $15 \mathrm{~mm}$ de longitud y $4 \mathrm{~mm}$ de diámetro, exceptuando los de la zona del 1.3. y 2.3. que eran de 3,25 mm. En los

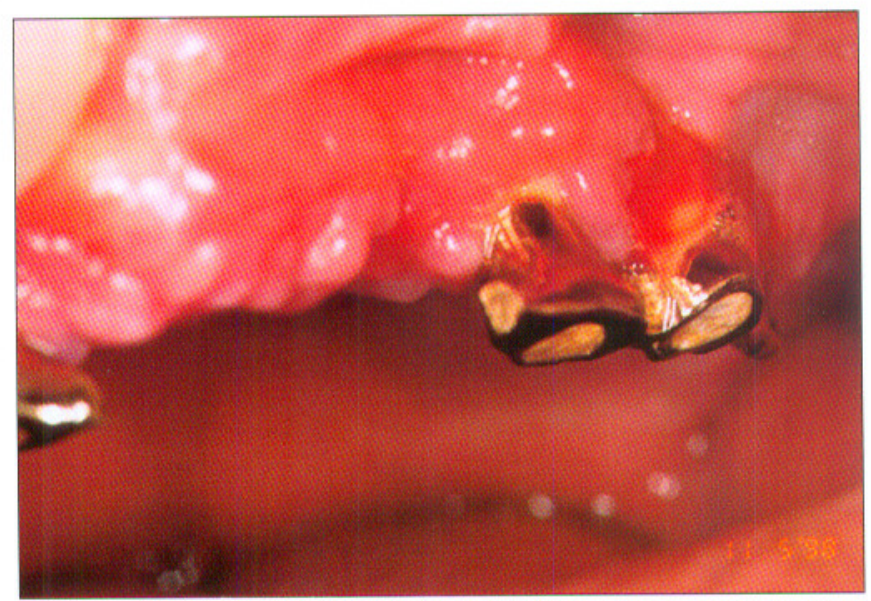

Fig. 3.: Caso 1: Supuración a nivel de los implantes 2.3 y 2.4.

implantes 1.3 y 2.3. se colocó una membrana reabsorbible de colágeno (Paroguide ${ }^{\circledR}$ ) con hueso autólogo obtenido durante el fresado de hueso para crear los lechos implantarios. La estabilidad inicial de los implantes fue excelente.

Seis meses más tarde, se realizó la segunda fase quirúrgica colocando 6 pilares de cicatrización y el control radiológico correspondiente. Se observó que el implante 1.1. presentaba una pérdida ósea circular de 3 milímetros y que las fenestraciones del 1.3. y del 2.3. no se habían regenerado. Posteriormente, se confeccionó una sobredentadura que tuvo que repetirse en tres ocasiones ya que se fracturaba en la línea media. A los siete meses de la segunda cirugía se observó una bolsa periimplantaria en el implante colocado en la posición del 1.1. y se decidió realizar una implantoplastia de la superficie del implante. No existía inflamación ni exudado de los tejidos blandos periimplantarios.

Tres años después de la colocación de los implantes, el paciente acudió de urgencia por presentar un absceso a nivel de la fosa canina izquierda que drenaba a nivel del implante mesial del 2.2. No presentaba movilidad ni dolor a la percusión. La radiología convencional mostró una imagen de discontinuidad a nivel del suelo de las fosas nasales. Se trató con 750 mg de amoxicilina y $150 \mathrm{mg}$ de clindamicina cada 8 horas durante 7 días. Una semana después se realizó la extracción del implante mesial del 2.2. y el legrado del absceso. Durante dicha intervención quirúrgica se observó que había producido una importante reabsorción ósea alrededor del implante.

Un año más tarde, durante una exploración de control, los implantes 2.3. y 2.4. presentaron unas bolsas periimplantarias de 3 a $8 \mathrm{~mm}$ de profundidad y presencia de supuración (figura 3). Se tomó una muestra del exudado perimplantario con puntas de papel estériles para realizar el estudio microbiológico 


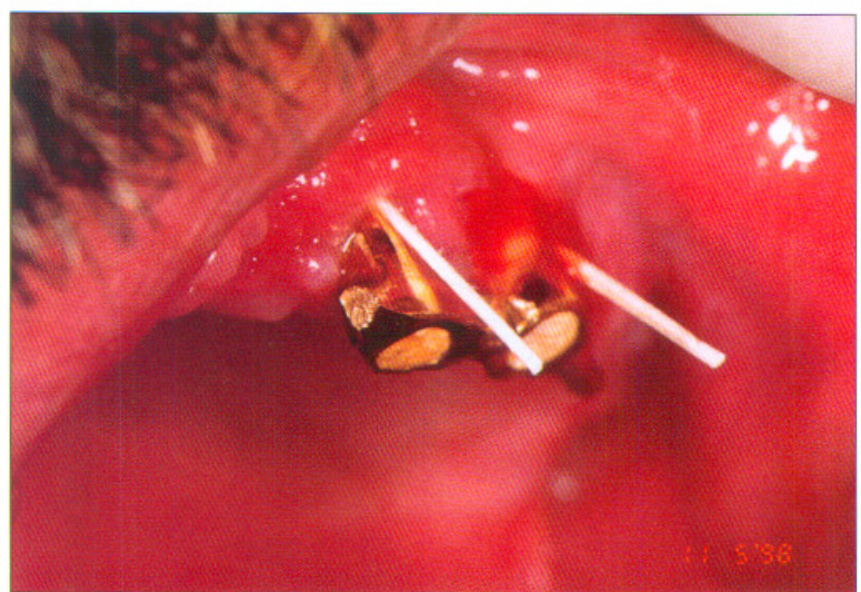

Fig. 4.: Caso 1: Obtención de muestras del exudado periimplantario con puntas de papel estériles.

(figura 4) y el resultado fue de Stomatococcus sensible a la combinación de amoxicilina y ácido clavulánico. Los dos implantes presentaban un tercio de las espiras expuestas. Se decidió realizar un curetaje con curetas de oro de la superficie de los implantes y una descontaminación con ácido cítrico al $40 \%$ durante 30 segundos. Sobre estos defectos óseos se colocó hueso bovino granulado (Bio-Oss ${ }^{\circledR}$ ) y una membrana reabsorbible de colágeno (BioMend ${ }^{\circledR}$ ). Como pauta antibiótica, el paciente tomó $875 \mathrm{mg}$ de amoxicilina y $125 \mathrm{mg}$ de ácido clavulánico por vía oral cada 8 horas durante 10 días y enjuagues con digluconato de clorhexidina al $0,12 \%$ dos veces al día durante 15 días. El postoperatorio fue bueno. Se replanteó el caso para poder realizar una nueva prótesis.

\section{CASO 2}

Paciente varón de 48 años de edad, sin antecedentes patológicos de interés y fumador de 25 cigarrillos al día que presentaba edentulismo total inferior y superior. Tras el estudio clínico-radiológico del caso se decidió colocar 6 implantes Screw-Vent ${ }^{\circledR}$ recubiertos de hidroxiapatita en el maxilar superior. En las posiciones del 1.3. y 2.3. se colocaron fijaciones de $16 \mathrm{~mm}$ de longitud, en las del 1.4. y 2.4. de $13 \mathrm{~mm}$ y en las del 1.5. y 2.5. de $10 \mathrm{~mm}$. El diámetro fue de $3,25 \mathrm{~mm}$ en todos los implantes. En la mandíbula se colocaron 5 implantes intermentonianos Screw-Vent ${ }^{\circledR}$ de $16 \mathrm{~mm}$ de longitud y de $3,25 \mathrm{~mm}$ de diámetro recubiertos de hidroxiapatita.

La prótesis mandibular se confeccionó a los tres meses de la colocación de los implantes y la prótesis del maxilar superior 12 meses después de la primera cirugía.

Transcurridos 4 meses de la colocación de la prótesis superior, el paciente presentó un exudado a nivel de la fijación 2.3. e inflamación de la mucosa periimplantaria. Se realizó el curetaje del implante 2.3. con curetas de oro, así como la descontaminación de su superficie con ácido cítrico al $40 \%$ durante 30 segundos. También se adelgazó la mucosa periimplantaria y se realizó un colgajo de reposición apical. Los controles anuales posteriores fueron correctos.

Tres años después se observó un exudado purulento a nivel de los implantes 2.4. y 2.5. El paciente no presentaba dolor pero radiológicamente existía una pérdida ósea en el 1.5, 1.4. y 1.3., así como en el 2.4. y el 2.5. Se extrajeron los implantes colocados en la posición del 1.5. y 2.5., ya que presentaban una pérdida ósea de más de dos tercios de su longitud. En el 1.3. y 1.4. se realizó la descontaminación con un curetaje abierto, desbridamiento y aplicación de ácido cítrico al $40 \%$ durante 30 segundos. A nivel del 2.4. se efectuó una plastia del implante, retirando la hidroxiapatita contaminada y colocando sobre la superficie metálica resultante polvo de clorhidrato de tetraciclina durante 5 minutos sin proceder a su lavado ni posterior remoción. La pauta antibiótica postoperatoria fue de $750 \mathrm{mg}$ de amoxicilina y $250 \mathrm{mg}$ de metronidazol, 3 veces al día por vía oral durante 16 días. El paciente también realizó enjuagues con digluconato de clorhexidina al $0,12 \%$. En el cultivo microbiológico que se realizó a partir de las muestras de los 6 implantes afectados no se encontró ninguna bacteria predominante.

Posteriormente, se reevaluó el diseño de la prótesis comprobando su ajuste pasivo y su correcta oclusión. Los controles clínicos y radiológicos anuales del paciente muestran una estabilidad de la pérdida ósea periimplantaria.

\section{CASO 3}

Varón de 61 años de edad, sin antecedentes patológicos de interés y fumador de 15 cigarrillos al día que presentaba edentulismo total superior e inferior. Tras realizar un estudio radiológico con una ortopantomografía y una teleradiografía lateral de cráneo, se decidió colocar tres implantes intermentonianos Brånemark $\mathrm{Mk}$ II de 3,75 mm de diámetro y $15 \mathrm{~mm}$ de longitud en las posiciones del 4.2., 3.1. y 3.3. En la zona del 3.3. se observó una dehiscencia vestibular en la que se colocó hueso autólogo resultante del fresado del lecho periimplantario y una membrana reabsorbible de colágeno (Bioguide ${ }^{\circledR}$ ). Se confeccionó una prótesis mucoimplantosoportada sobre bolas. Cuatro años después se observó un fracaso del implante 3.3. que fue extraído. La encía periimplantaria presentaba un aspecto hipertrófico y la higiene bucal del paciente era muy deficiente. El estudio microbiológico del tejido de granulación periimplantario no pudo determinar ninguna microflora predominante. 


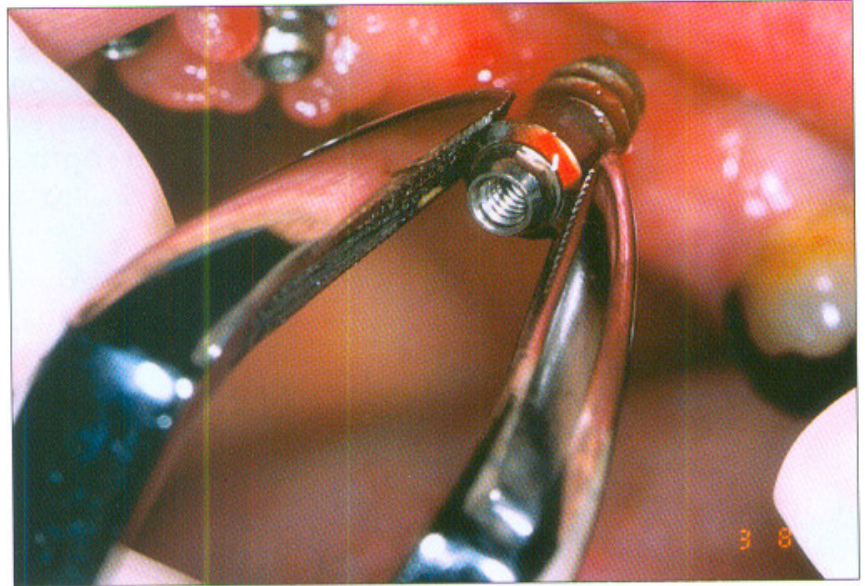

Fig. 5.: Caso 5: Extracción del implante 2.5. con fórceps.

\section{CASO 4}

Varón de 40 años de edad, sin antecedentes patológicos de interés, no fumador, que presentaba edentulismo total inferior. En la primera fase quirúrgica se colocaron tres implantes intermentonianos Calcitek Integral ${ }^{\circledR}$ de $15 \mathrm{~mm}$ de longitud y $3,25 \mathrm{~mm}$ de diámetro. El postoperatorio a los siete días fue normal. Cuatro meses después se realizó la segunda fase quirúrgica y se observó una falta de encía queratinizada alrededor de los pilares de cicatrización así como una higiene bucal muy deficiente. A los cinco años de la colocación de los implantes se apreció una periimplantitis a nivel de los implantes colocados en las posiciones 4.1. y 4.3. en los que se había producido una pérdida ósea periimplantaria de dos tercios de su longitud. El paciente continuaba con mala higiene bucal. Estos dos implantes presentaban una movilidad importante y se decidió su extracción. El cultivo microbiológico mostró la presencia de Fusobacterium nucleatun sensible a la asociación de amoxicilina y ácido clavulánico.

\section{CASO 5}

Mujer de 45 años de edad, sin antecedentes patológicos de interés que presentaba edentulismo total del maxilar superior y era bruxista céntrica. Como pruebas complementarias se indicaron una ortopantomografía y una tomografía computadorizada del maxilar superior. Se colocaron 6 implantes macizos roscados ITI $^{\ominus}$ en las posiciones 1.5., 1.3., 1.2., 2.2., 2.3. y 2.5. Durante la primera fase se observó una dehiscencia de $3 \mathrm{~mm}$ a nivel de los implantes 1.2. y 2.2. En la segunda fase se vió que el implante 1.2. presentaba una movilidad tipo II y se decidió su extracción. A continuación, se tomaron las impresiones para realizar una prótesis mucoimplantosoportada. Se elaboró una férula de descarga que la paciente no utilizó, por

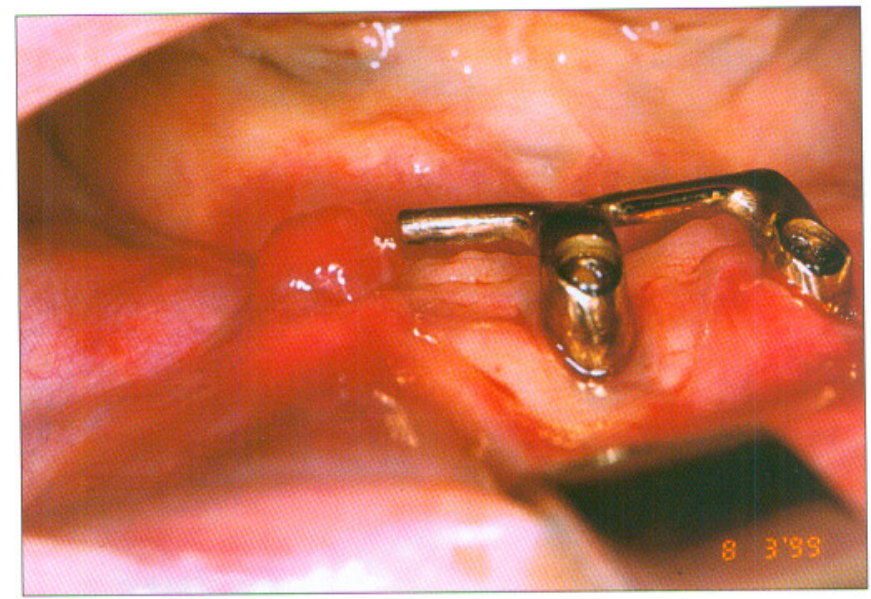

Fig. 6.: Caso 6: Hipertrofia gingival en el implante situado en la posición del 4.3. La barra que unía los tres implantes ya había sido seccionada.

lo que se tuvo que confeccionar la prótesis en tres ocasiones debido a que las fracturaba en su línea media.

Cinco años más tarde, las radiografías de control mostraron una pérdida ósea vertical en el implante 2.5. Se desmontó la supraestructura y se comprobó la movilidad del implante y la presencia de exudado. Con puntas de papel estériles se tomaron muestras y se extrajo el implante (figura 5 ). El resultado microbiológico mostró que no existía ninguna especie bacteriana predominante. Se trató satisfactoriamente con la asociación de la amoxicilina con ácido clavulánico. Se tuvo que volver a elaborar la prótesis y reevaluar el caso para repartir correctamente las fuerzas de la oclusión.

\section{CASO 6}

Mujer de 63 años de edad, hipertensa controlada farmacológicamente, que fue remitida a nuestro servicio para realizar una evaluación del caso y la colocación de una prótesis nueva sobre sus implantes. La paciente presentaba edentulismo total superior e inferior. Hacía tres años que le habían colocado tres implantes intermentonianos de la firma Klockner ${ }^{\oplus}$. Sobre estos implantes se colocaron dos caballetes y una barra de Doler que soportaba una sobredentadura implantomucosoportada. El implante 4.3. presentaba una movilidad tipo II, exudado purulento y una hipertrofia gingival (figura 6). Con anestesia local se realizó la extracción del implante 4.3., previo estudio microbiológico del exudado periimplantario. La bacteria predominante fue un Peptostreptococcus que resultó sensible a la asociación de amoxicilina con ácido clavulánico. A pesar de la movilidad del implante, la extracción se reali- 


\section{AVANCES}

Volumen 12 - No 3 - Diciembre 2000

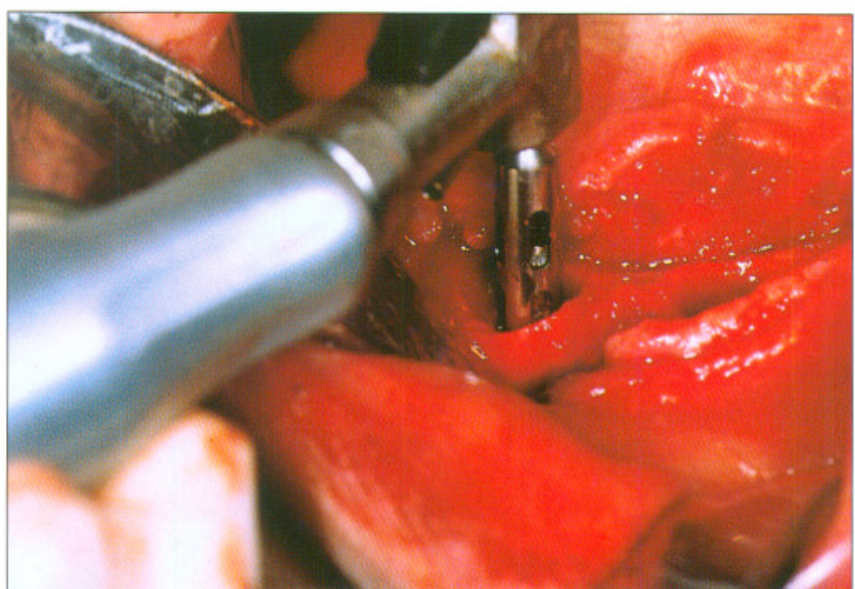

Fig. 1.: Caso 6: Extracción del implante 4.3. utilizando una trefina de hueso.

zó con una trefina de hueso (figura 7). El control postoperatorio a los siete días fue correcto. Al cabo de dos semanas se modificó su prótesis implantomucosoportada, que actualmente, se apoya sobre una barra que une los dos implantes intermentonianos restantes.

\section{CASO 7}

Mujer de 18 años de edad, sin antecedentes patológicos de interés y portadora de ortodoncia fija durante tres años para crear el espacio suficiente y colocar dos implantes en la posición del 1.2. y 2.2. que estaban ausentes debido a una agenesia. En la primera fase quirúrgica se utilizaron dos fijaciones Steri-Oss ${ }^{\circledR}$, de $16 \mathrm{~mm}$ de longitud y 3,25 mm de diámetro en las posiciones 1.2. y 2.2. A los seis meses se colocaron las dos coronas implantosoportadas. En los controles anuales posteriores se observó un ligero enrojecimiento de la encía periimplantaria del 2.2. y se insistió en la importancia de mantener una buena higiene bucal. Tres años más tarde se apreció una recesión gingival de $3 \mathrm{~mm}$ a nivel del implante localizado en la posición del 2.2. y la salida de un exudado mucopurulento cuando se realizaba una presión sobre la encía periimplantaria. A continuación, se retocó la oclusión de la corona del implante 2.2. y se realizó el cultivo del exudado. Bajo anestesia local se levantó un colgajo triangular de espesor completo con descarga en el 2.3., se eliminó el tejido de granulación periimplantario y se colocó tetraciclina en polvo en la superficie del implante (figura 8), suturando a continuación con seda. El control postoperatorio a los siete días fue normal. Los controles realizados cada seis meses hasta ahora muestran una evolución favorable. El resultado del cultivo fue de Stomatococcus mucilaginosus sensible a la asociación de la amoxicilina con el ácido clavulánico.

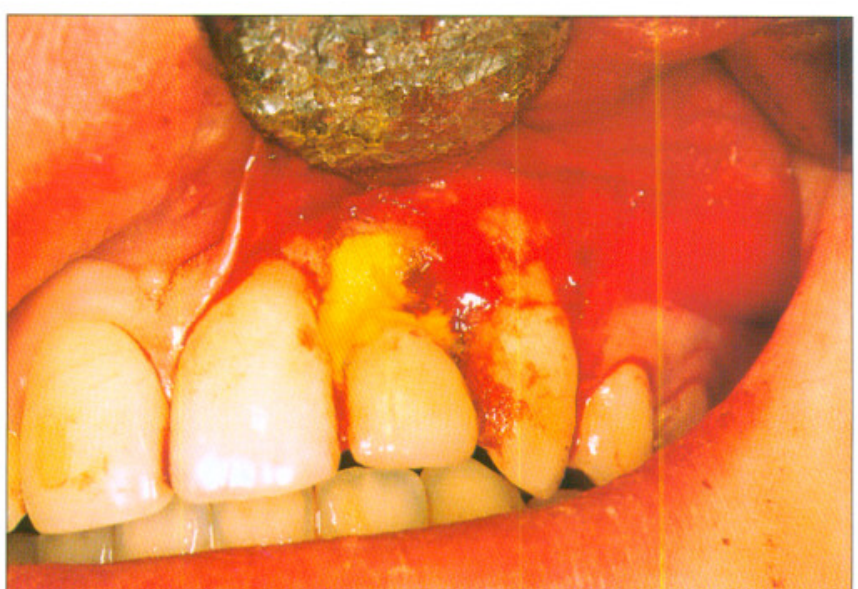

Fig. 8.: Caso 7: Aplicación de tetraciclina en polvo en el implante en la posición 2.2. para descontaminar la superficie del mismo.

\section{CASO 8}

Varón de 67 años de edad, exfumador desde hace 1 año y diabético insulinodependiente, que presentaba un estadío muy avanzado de enfermedad periodontal en los dientes localizados en el maxilar superior. Se decidió realizar la extracción de los 1.2., 1.1., 2.1., 2.2., 2.3. y 2.6. y colocar una prótesis completa superior inmediata. Dos meses después se realizó la primera fase quirúrgica con la colocación de 5 implantes Brånemark. Las fijaciones eran estándar de 3,75 mm de diámetro cuyas longitudes fueron de $8,5 \mathrm{~mm}$ en la posición del 1.4., de $15 \mathrm{~mm}$ en la del 1.3. y de $13 \mathrm{~mm}$ en las posiciones del 1.2., 2.3. y 2.4. Se observó una fenestración apical del implante 1.3. y en el tercio medio del 2.3. La estabilidad primaria fue buena y el postoperatorio a los siete días fue correcto. Diez meses más tarde se colocó una sobredentadura. Durante el control anual se observó la presencia de una fístula en el implante 2.4., con una tumefacción y enrojecimiento de la encía periimplantaria, acúmulo de placa pero sin signos de movilidad. Radiológicamente, se comprobó que se había producido una pérdida de hueso vertical periimplantaria en el implante 2.4. Se tomó una muestra con puntas de papel estériles del surco periimplantario para realizar el estudio microbiológico. Como resultado se observó que la especie predominante era Prevotella oralis, destacando también que un $20 \%$ de los gérmenes eran Prevotella melaninogenica. La Prevotella oralis era sensible a la asociación de la amoxicilina con el ácido clavulánico. El tratamiento realizado fue la descontaminación de la superficie del implante a cielo abierto con ácido cítrico $(\mathrm{pH}=1,40 \%$ de concentración) durante 1 minuto, la colocación de antibiótico local (tetraciclina en polvo) y antibioticoterapia sistémica con $875 \mathrm{mg}$ de amoxicilina con $125 \mathrm{mg}$ de ácido clavulánico por vía oral cada 8 horas durante 15 días. Los controles postoperatorios sucesivos mostraron una buena evolución del implante. En el control 
realizado dos años después del tratamiento, no se ha observado ningún cambio patológico, ni signos de periimplantitis. La higiene bucal del paciente es correcta, el sondaje del 2.4. no supera en ningún punto los $2 \mathrm{~mm}$ de profundidad y los controles radiológicos muestran que se mantiene el nivel óseo.

\section{CASO 9}

Varón de 50 años de edad, hipertenso controlado farmacológicamente y fumador de 20 cigarrillos al día, que presentaba edentulismo total en el maxilar superior. Se decidió colocar 6 implantes Microvent ${ }^{\text {i de }}$ titanio recubiertos con hidroxiapatita de $3,25 \mathrm{~mm}$ de diámetro. En las posiciones 1.3., 2.1. y 2.3. fueron de $13 \mathrm{~mm}$ de longitud y de $16 \mathrm{~mm}$ en el 1.4, 1.1. y 2.4 . Seis meses más tarde se colocó una sobredentadura implantomucosoportada. Tres años después de la primera cirugía, el paciente acudió a nuestro servicio por presentar un exudado en los implantes 2.3. y 2.5. El cultivo de este exudado no mostró ninguna bacteria predominante y se trató satisfactoriamente con la asociación de amoxicilina con ácido clavulánico. El tratamiento realizado consistió en la extracción del implante de la posición 2.3. y la descontaminación de la superficie del 2.5. con ácido cítrico ( $\mathrm{pH}=1,40 \%$ de concentración) durante 30 segundos. En los controles posteriores se ha observado un buen estado de la encía que rodea el implante y radiológicamente los niveles óseos periimplantarios se mantienen estables al año del tratamiento.

\section{DISCUSIÓN}

Las bacterias presentes en las periimplantitis tratadas en nuestro estudio fueron: Stomatococcus, Stomatococcus mucilaginosus, Fusobacterium nucleatum, Peptostreptococcus y Prevotella oralis. En ningún caso se encontraron cepas de Actinobacillus actinomycetemcomitans. Todas las bacterias aisladas mostraron una gran sensibilidad a la asociación de amoxicilina y ácido clavulánico, siendo menos sensibles a la amoxicilina, al metronidazol o bien a la combinación de amoxicilina y metronidazol.

En cuanto al tratamiento protésico realizado, tres pacientes eran portadores de sobredentaduras mandibulares implantomucosoportadas; en estos casos se tuvo que repetir la confección de la sobredentadura en varias ocasiones, ya que se fracturaba a nivel de la línea media. En cinco pacientes la rehabilitación protésica fue una sobredentadura en el maxilar superior y los implantes afectados de periimplantitis fueron los colocados en la posición más distal de la prótesis, en la zona más posterior de los extremos libres edéntulos. Sólo en el caso 7 se trató de un implante unitario.

En relación a la patología periodontal destacamos que 8 de los 9 pacientes tratados presentaban antecedentes de enfermedad periodontal. Cuatro pacientes eran edéntulos totales por esta causa y los otros cuatro presentaban edentulismo parcial con enfermedad periodontal en los dientes remanentes. Sólo hubo un caso sin antecedentes de enfermedad periodontal.

En cuanto a la superficie del implante, 13 de los 16 implantes tratados estaban recubiertos de hidroxiapatita y tenían un diámetro de 3,25 mm.

Los tejidos blandos que rodean al implante son un mecanismo de protección y actúan como barrera biológica ante los posibles agentes etiológicos de la enfermedad periimplantaria7. La pérdida del sellado mucoso periimplantario, combinado con un deficiente control de placa, facilita la proliferación de bacterias anaeróbicas que penetran en el surco periimplantario produciendo mucositis. Si esta situación no se trata adecuadamente, pasados 10-15 días, puede evolucionar a una periimplantitis con pérdida de hueso periimplantario ${ }^{12}$.

Según Exposito y cols. ${ }^{13}$, la periimplantitis se define como una infección localizada, inducida por la placa bacteriana y con una pérdida progresiva de soporte óseo alrededor de un implante funcional. La periimplantitis se inicia en la porción coronal del implante, mientras que en la porción más apical el implante se mantiene oseointegrado ${ }^{14}$.

Los microorganismos predominantes en la cavidad bucal en estado de salud son los cocos Gram positivo y los microorganismos no móviles ${ }^{7}$. Parece ser que la flora subgingival y periimplantaria que presenta el diente y el implante sano es bastante similar ${ }^{16,16}$. Los estudios microbiológicos de la zona periimplantaria en implantes con éxito muestran, generalmente, bajas cantidades de anaerobios y altas cantidades de cocos y bacilos aerobios mientras que los implantes fallidos presentan un alto número de anaerobios Gram negativo y espiroquetas $\mathbf{s}^{5,17-19}$.

En nuestro estudio se observó que los especimenes asociados a la periimplantitis eran Stomatococcus, Fusobacterium nucleatum, Prevotella oralis y Peptostreptococcus. Estos resultados concuerdan con los hallazgos de otros estudios ${ }^{5,9,17,20-23}$, donde se hallaron preferentemente bacilos Gram negativo anaerobios estrictos como Fusobacterium spp y/o Prevotella.

El Stomatococcus es un coco Gram positivo anaerobio facultativo o microaerófilo. En la flora bucal el 
Stomatococcus mucilaginosus es una bacteria saprófita; sin embargo, en abscesos odontogénicos actúa como patógena. Dentro de los anaerobios estrictos Gram positivo no móviles encontramos al Peptostreptococcus, que participa en los abscesos de origen odontogénico. Los Bacteroides y Fusobacterium son anaerobios estrictos Gram negativo no móviles. Algunos Bacteroides, que se han reclasificado actualmente dentro del género Prevotellas, tales como $P$. melaninogenia y $P$. Oralis, están asociados con la enfermedad periodontal ${ }^{24-26}$.

Es típico que las infecciones anaerobias sean polimicrobianas. Bacteroides, Prevotella, Porphyromonas y Fusobacterium actúan, especialmente, en la destrucción tisular y en la formación de abscesos. Su multiplicación se produce muy rápidamente y mantienen el proceso destructivo, no sólo por la presencia y cantidad de bacterias, sino por las toxinas que van eliminando al medio y la respuesta que producen en el hospedador. Son un factor importante en la formación y maduración de la placa bacteriana ${ }^{27}$. Se cree que estas infecciones polimicrobianas producidas por anaerobios potencian la sinergia entre las diferentes especies bacterianas ${ }^{9,26}$. La proliferación de diversas cepas anaeróbicas explicaría que no se pudiera determinar cuál era el microorganismo predominante en algunos casos.

A nivel experimental, Eke y cols. ${ }^{28}$ realizaron un trabajo sobre la microflora de implantes funcionales en monos parcialmente edéntulos. A todos los animales se les realizaba una profilaxis cada mes. Se hicieron valoraciones microbiológicas desde la primera cirugía implantológica hasta seis meses después de haber colocado la prótesis implantosoportada. En las primeras mediciones se encontraron cepas de Prevotella intermedia y Prevotella melaninogenica. Tres meses después de haber colocado la prótesis, los niveles de Prevotella intermedia y A. actinomycetemcomitans habían aumentado, pero a los seis meses de llevar la prótesis, estos niveles habían disminuido significativamente. Por otro lado, las bolsas periimplantarias en este tiempo se asociaban a la presencia de Prevotella intermedia, Porphyromonas spp y espiroquetas $^{28}$. Sin embargo, en nuestro estudio, y coincidiendo con Sbordone y cols. ${ }^{20}$, no hemos hallado cepas de Actinobacillus actinomycetemcomitans en ningún caso.

En 1987, Mombelli y cols. ${ }^{5}$ presentaron un estudio sobre la microflora de 7 implantes que habían fracasado en 7 pacientes. Clínicamente, observaron una pérdida ósea vertical marcada ( $\geq$ a $6 \mathrm{~mm}$ ) y una supuración de la zona periimplantaria. Se emplearon puntas de papel estériles para la obtención de muestras a nivel sulcular y se realizaron cultivos anaerobios y aerobios igual que en nuestro estudio. Los resultados reflejaron que un $41 \%$ de los microorganismos cultivados fueron bacilos anaerobios Gram negativo, como por ejemplo Fusobacterium, que también ha sido hallado en una de nuestras muestras, y Prevotella intermedia. Se compararon los resultados con muestras microbiológicas de los implantes oseointegrados con éxito. La flora bacteriana presente en los surcos periimplantarios de estos implantes sanos estaba compuesta básicamente por cocos Gram positivo. A partir de estos hallazgos se postuló la teoría sobre los microorganismos asociados a la periimplantitis estableciendo que las bacterias de la periimplantitis eran las mismas que las que producían la periodontitis crónica en los dientes naturales. Estos resultados sugieren que los dientes naturales son una importante fuente de bacterias para la colonización de los implantes ${ }^{7}$.

Algunos autores describen una marcada diferencia entre la microflora presente en la periimplantitis de pacientes totalmente edéntulos y de los parcialmente edéntulos ${ }^{6,29-31}$. Los patógenos periodontales se encuentran en menor cantidad en los surcos periimplantarios de los individuos totalmente edéntulos ${ }^{6}$. Un estudio longitudinal en pacientes realizado por Mombelli y cols. ${ }^{17}$ sobre la evolución de la microflora asociada a implantes con infección periimplantaria mostró que se producía un aumento progresivo de Fusobacterium. Estas bacterias eran detectables a los 42 días de la colocación del implante. A partir del día 21 , los cocos habían disminuido y aumentaban los bacilos. Pasados 120 días se podían encontrar espiroquetas, supuración de exudado y bolsas periimplantarias de más de $6 \mathrm{~mm}$. Este estudio se llevó a cabo en pacientes edéntulos totales y las muestras se tomaban semanalmente de la bolsa periimplantaria ${ }^{17}$. Por otro lado, Leonhardt y cols..$^{23}$ realizaron un estudio longitudinal sobre la microflora de la periimplantitis en pacientes parcialmente edéntulos, concluyendo que, a pesar de que los patógenos de la enfermedad periodontal pueden colonizar rápidamente la superficie de los implantes, no significa que el implante fracase necesariamente. En nuestro trabajo sólo había un paciente que no tenía antecedentes de enfermedad periodontal (caso 7) donde hallamos especímenes de Stomatococcus mucilaginosus. En el resto de pacientes existían antecedentes de enfermedad periodontal. Según nuestro estudio, en los pacientes parcialmente edéntulos y con antecedentes de patología periodontal, encontramos especímenes de Prevotella oralis y Stomatococcus, coincidiendo con Eke y cols. ${ }^{28}$ en el hallazgo de Prevotellas. Sin embargo, otros estudios muestran más cepas de anaerobios Gram negativo, menos cocos y más espiroquetas alrededor de los implantes en pacientes edéntulos parciales que en totalmente edéntulos ${ }^{29,30}$. Por otro lado, en los pacientes totalmente edéntulos cuya causa de la pérdida dentaria fue la enfermedad periodontal, 
hallamos Fusobacterium nucleatum y Peptostreptococcus. Esto concuerda con los hallazgos previos de Mombelli y cols. ${ }^{17}$, que refieren la asociación de Fusobacterium y la periimplantitis en estos pacientes.

Hultin y cols. ${ }^{18}$ evaluaron la flora bacteriana de 30 pacientes que acudían a controles anuales de sus implantes, sin presentar ningún problema de tipo periimplantario. Investigaron la respuesta inflamatoria mediante índices gingivales y la concentración de proteínas presente en los tejidos gingivales y periimplantarios de implantes sanos y dientes naturales. La actividad de los neutrófilos fue mayor alrededor de los implantes de los pacientes parcialmente edéntulos que en los totalmente edéntulos. Talarico y cols. ${ }^{32}$ estudiaron la respuesta inflamatoria de los tejidos blandos que rodean a un implante $o$ a un diente en una situación de periimplantitis o periodontitis. Los resultados fueron similares en las dos entidades y se observó la participación del mismo tipo de células inflamatorias en los dos procesos. Por lo tanto, el tratamiento de los tejidos de alrededor de un implante con periimplantitis para reducir la inflamación periimplantaria y el acúmulo de placa será de gran utilidad para controlar la periimplantitis, igual que en la enfermedad periodontal.

La detección precoz de zonas potenciales a padecer enfermedad periodontal o periimplantaria es difícil. Oringer y cols.$^{21}$ diseñaron un estudio sobre el piridinoline telopeptido carboxiterminal (ICTP) de enlace cruzado de colágeno tipo I, un marcador específico de la degradación ósea encontrado en el fluido crevicular gingival, que se ha asociado con la pérdida de hueso en la periodontitis. El objetivo de esta investigación era determinar los niveles de ICTP y las especies microbianas presentes en los surcos de los implantes y los dientes naturales en 22 pacientes. En la mayoría de casos se trataba de pacientes parcialmente edéntulos. Los niveles de ICTP y la composición de la placa no fueron significativamente diferentes entre los dientes naturales y los implantes. Los lugares con niveles más elevados de ICTP se asociaron a implantes o dientes con pérdida de soporte óseo vertical. En estos lugares las especies encontradas fueron Prevotella intermedia, Capnocytophaga gingivalis, Fusobacterium nucleatum ss vicentii, y Streptococcus gordinii ${ }^{21}$. Pero no debemos olvidar que la actividad y virulencia de todos estos microorganismos también viene determinada por la respuesta del hospedador ante la presencia bacteriana que permite que estas bacterias sean oportunistas o $\mathrm{no}^{8,22}$.

Algunas investigaciones en perros ${ }^{33}$ demuestran que la combinación de la amoxicilina con el metronidazol es apropiada para el tratamiento de las lesiones periimplantarias con afectación de la mucosa y el hueso. Se comprobó que la terapia antimicrobiana sistémica durante tres semanas combinada con el curetaje a cielo abierto de la superficie del implante era positiva para la resolución de la lesión periimplantaria ${ }^{33}$. Se obtuvieron los mismos resultados en otro estudio realizado por Persson y cols..$^{34}$ en perros beagles. En este estudio se administró la misma asociación antimicrobiana y se instauraron medidas de higiene local pero no se realizó ningún tratamiento quirúrgico. La asociación de la amoxicilina y el metronidazol para el tratamiento de la periimplantitis derivó del protocolo del tratamiento de la periodontitis refractaria contra Actinobacillus actinomycetemcomi$\tan s^{36}$.

Sbordone y cols. ${ }^{20}$ investigaron la susceptibilidad antibiótica de los microorganismos asociados a 19 implantes fracasados en un total de 13 pacientes. Las bacterias más frecuentes eran anaerobios Gram negativo, destacando Fusobacterium nucleatum, Prevotella intermedia y Porphyromonas gingivalis. Los antibiogramas mostraron que Porphyromonas gingivalis y Prevotella intermedia eran más sensibles a la penicilina G y a la amoxicilina. Sin embargo, Fusobacterium nucleatum fue más sensible a la amoxicilina y a la asociación de la amoxicilina con el metronidazol. Ninguno de los microorganismos estudiados mostró resistencia a la penicilina $\mathrm{G}$, a la amoxicilina, a la asociación amoxicilina con ácido clavulánico o a la combinación de la amoxicilina con el metronidazol. La clindamicina y las tetracilinas no se mostraron efectivas ante estos microorganismos y la eritromicina y el metronidazol eran ligeramente activos ante las bacterias $^{20}$. En nuestros antibiogramas todas las bacterias fueron más sensibles a la asociación de amoxicilina con ácido clavulánico que a la amoxicilina o a la combinación de amoxicilina con metronidazol.

Otro factor muy importante en la aparición de la periimplantitis es la sobrecarga oclusal. El estrés biomecánico asociado a las fuerzas de la masticación dan lugar a la pérdida de hueso periimplantario. Posteriormente, el surco periimplantario se coloniza y se infecta por microorganismos ${ }^{36}$. El papel de la sobrecarga biomecánica en el origen de la periimplantitis es mayor cuando el implante está colocado en una posición o inclinación incorrecta, si existen parafunciones o si el número total de implantes no es el adecuado para una correcta distribución de las fuerzas masticatorias ${ }^{4,37}$. La aparición de estrés oclusal y de microfracturas a nivel de la porción coronal del implante por fuerzas biomecánicas excesivas conlleva la pérdida de hueso a nivel periimplantario, existiendo de todo ello evidencias clínicas y experimentales $^{38,39}$.

Rosenberg y cols. ${ }^{9}$ realizaron un estudio sobre los microorganismos que colonizaban los implantes con periimplantitis, tanto de origen infeccioso como por 
sobrecarga oclusal. Si la periimplantitis era de origen infeccioso, la clínica era muy marcada con supuración, índices de placa elevados, sangrado, etc., y presentaban especimenes de espiroquetas y bacterias del tipo Peptostreptococcus y Fusobacterium. En cambio, las periimplantitis por sobrecarga biomecánica mostraban una microflora mayoritariamente compuesta por Streptococcus y microorganismos no periodontopatógenos.

Debe valorarse la ubicación de los implantes ya que es frecuente que exista un mayor índice de fracasos en la región posterior del maxilar superior y de la mandíbula debido a que el hueso es más blando y a que las fuerzas de la masticación son mayores en estas zonas ${ }^{40-42}$. En nuestra serie destacamos que en cinco pacientes los implantes con periimplantitis estaban colocados en las posiciones más distales de sobredentaduras superiores, donde la fuerza de palanca sobre los implantes es mayor si la prótesis no está correctamente confeccionada.

El control de placa bacteriana parece ser más importante en los implantes dentales que en los dientes naturales ${ }^{43}$. La placa subgingival está compuesta básicamente por bacterias Gram negativo y flora anaerobia, que son el principal factor etiológico de la enfermedad periodontal y de la periimplantitis. Sobre la flora de la periimplantitis, algunos autores ${ }^{5}$ hablan de determinadas especies como Actinobacillus actinomycetemcomitans, Porphyromonas gingivalis y Prevotella intermedia, esta última muy frecuente en la enfermedad periodontal ${ }^{44}$. La mayoría de nuestros pacientes no presentaban un buen control de placa, bien sea por falta de higiene bucal o por un mal diseño de la prótesis. Por otra parte, en implantes sin encía queratinizada es importante mantener una buena higiene bucal para evitar posibles complicaciones como la periimplantitis ${ }^{7}$.

La capacidad de adherencia de las bacterias a la superficie del implante dental, sea titanio o hidroxiapatita, es un factor determinante para garantizar el éxito del mismo. Algunos estudios ${ }^{42}$ comprueban clínicamente la velocidad de reabsorción ósea a nivel periimplantario en implantes recubiertos con hidroxiapatita. Como explican Slaughter y cols. ${ }^{45}$, la hidroxiapatita sufre una disolución y degradación celular que favorece la colonización por parte de los microorganismos oportunistas, condicionando una respuesta clínica desfavorable.

En 1998, Ichikawa y cols ${ }^{46}$. realizaron un estudio in vitro para valorar la adherencia bacteriana a la hidroxiapatita y al titanio. Emplearon cepas de Streptococcus constellatus y fabricaron cilindros de $2 \mathrm{~mm}$ de diámetro por $5 \mathrm{~mm}$ de largo de cada material (titanio e hidroxiapatita). La hidroxiapatita se presentaba en tres formas: tratada con ácido fluorhídrico, en contacto con la saliva humana o sin ningún tratamiento externo. La adherencia de los microorganismos a la superficie no tratada de la hidroxiapatita fue dos veces más alta que la del titanio. El contacto con la saliva aumentaba la adherencia de las bacterias a la superficie del material. Existía una relación exponencial entre el tiempo de contacto con la saliva y el grado de adherencia bacteriana. Estos autores comprobaron que una mayor rugosidad de la superficie como en los implantes recubiertos por hidroxiapatita es más susceptible a la colonización microbiana. Además, creen que el acúmulo de placa será mayor en una superficie de hidroxiapatita expuesta al medio bucal que en una de titanio. Por lo tanto, se debe investigar más sobre la superficie del implante para facilitar su oseointegración y prevenir la colonización bacteriana ${ }^{47}$.

Por otro lado, Tillmanns y cols. ${ }^{8}$ realizaron un estudio en perros beagles donde demostraron que no existen diferencias significativas en la presencia de microorganismos en la hidroxiapatita, el plasma de titanio y la aleación de titanio como superficies implantarias. En este estudio se valoraban las muestras desde un punto de vista radiológico, además de la histología periimplantaria y el estudio microbiológico del surco periimplantario basado en el ADN bacteriano. También se emplearon puntas de papel estériles en esta investigación. En nuestro estudio, 13 de los 16 implantes tratados estaban recubiertos de hidroxiapatita y tenían un diámetro de 3,25 mm.

Un factor que no se ha tenido en cuenta en nuestro estudio pero que sería interesante valorar es la calidad de hueso del paciente. Algunos autores ${ }^{40,45}$ presentan resultados en los que se comprueba que el hueso tipo IV, según la clasificación de Brånemark ${ }^{16}$, presenta un mayor número de fracasos.

El mejor tratamiento de la periimplantitis es su prevención. Esto se consigue siendo muy rigurosos durante las fases quirúrgica y protésica. Hemos de realizar previamente un diagnóstico y plan de tratamiento correctos para determinar el número y la posición ideal de los implantes, según las características y posibilidades de cada paciente. El diseño de la prótesis debe presentar un ajuste pasivo y permitir una buena higiene bucal y un buen control de la placa bacteriana. Si el paciente presenta alguna parafunción debe tratarse previamente ${ }^{48-51}$. También es muy importante efectuar controles periódicos de los pacientes, tanto clínica como radiológicamente ${ }^{50}$.

Cuando la periimplantitis ya se ha instaurado, lo más importante es realizar su diagnóstico precoz para que nuestro tratamiento tenga mayores posibilidades de éxito. Su tratamiento se basa en consideraciones 
empíricas derivadas de investigaciones sobre la enfermedad periodontal. Algunos autores como Salcetti y cols ${ }^{52}$ han investigado sobre la administración sistémica de los antiinflamatorios no esteroideos (AINEs) como tratamiento en la prevención de la pérdida de la cresta ósea periimplantaria tras la colocación de los implantes dentales. De esta manera se evitaría la respuesta inflamatoria del hospedador producida por un aumento en los niveles de los mediadores antiinflamatorios que es uno de los factores más importantes en la etiología de la enfermedad periodontal y de la periimplantitis ${ }^{53}$. En un futuro, se deberá estudiar más sobre la aplicación, como tratamiento precoz de la periimplantitis, de los AINEs tópicos para disminuir o eliminar los efectos secundarios que producen cuando son administrados de forma sistémica. Salvi y cols. ${ }^{53}$ postulan que la aplicación de los nuevos AINEs con tarjetas especiales para la cicloxigenasa-2, en combinación con agentes antimicrobianos, pueden proporcionar nuevas estrategias en el tratamiento local de la enfermedad periodontal y de la periimplantitis.

El tratamiento de la periimplantitis dependerá de la cantidad de hueso periimplantario remanente y la movilidad del implante. Si la pérdida ósea es incipiente y existe supuración el tratamiento consiste en desmontar la prótesis y prescribir antibióticos y antiinflamatorios no esteroideos. Si la pérdida ósea es avanzada $\circ$ persistente se necesitará recurrir a la cirugía para desbridar los tejidos blandos periimplantarios originados por la infección, descontaminando la superficie del implante y realizar técnicas de regeneración ósea guiada si el defecto lo precisa ${ }^{7}$. Dependiendo de la morfología y el tamaño de la lesión ósea las técnicas quirúrgicas serán resectivas o regenerativas. Si la afectación ósea es de pocos milímetros se efectuarán técnicas resectivas como en el caso 7. Por otra parte, si la pérdida ósea es severa se recurrirá a las técnicas regenerativas con la descontaminación previa de la superficie del implante y la utilización de técnicas de regeneración ósea guiada ${ }^{4}$ como en el caso 1 .

La relación entre la superficie del implante y los tratamientos de descontaminación fue estudiada in vitro por Dennison y cols. ${ }^{54}$ que llegaron a la conclusión que el arenado usando sistemas abrasivos aire-polvo (suero fisiológico y bicarbonato), es el mejor sistema para eliminar endotoxinas en todas las superficies (hidroxiapatita, plasma de titanio o superficie de titanio sin recubrimiento) y que el ácido ć́trico a un $40 \%$ de concentración y pH l colocado en la superficie del implante durante 30-40 segundos es un método efectivo sobre implantes recubiertos de hidroxiapatita, como hemos efectuado en nuestros casos $1,2,8$ y 9 . Por otra parte, estos autores explican que la clorhexidina es ineficaz para eliminar endotoxinas de la superficie de la hidroxiapatita y que la superficie de titanio sin materiales de recubrimiento es la que se descontamina mejor ${ }^{11,54}$.

No se ha identificado ninguna enfermedad sistémica que contraindique de forma absoluta la cirugía implantológica. Como contraindicaciones relativas se incluyen la diabetes, la irradiación, enfermedad cardíaca y los tratamientos de larga duración con corticosteroides ${ }^{7}$. No hemos encontrado artículos que hablen de la relación entre enfermedades sistémicas y el riesgo de padecer periimplantitis. Dos de nuestros pacientes eran hipertensos y uno diabético.

En pacientes fumadores existe un mayor número de periimplantitis que en los no fumadores ${ }^{56}$, ya que el tabaco actúa como irritante local e interfiere en el sistema inmunitario de defensa, lo que agrava cualquier tipo de lesión periodontal o periimplantaria. En este trabajo cuatro pacientes eran fumadores de más de 15 cigarrillos al día y dos eran exfumadores. También existen estudios que comprueban un mayor riesgo de fracaso en los implantes colocados en el maxilar superior en pacientes fumadores ${ }^{56}$, como sucede en cuatro pacientes de nuestro estudio.

\section{ABSTRACT}

Objective: Peri-implantitis is an inflammatory process affecting the tissues around an osseointegrated implant in function, resulting in loss of supporting bone. The aim of this study was to evaluate the subgingival microbiota at dental implants associated with peri-implantitis treated in our service. A total of 16 implants of 9 patients were studied. In all cases implants were under functional conditions.

Materials and Methods: We studied 16 peri-implantitis in 9 patients; in each patient subgingival bacterial samples were obtained using paper-points or inflammatory soft tissue, and subjected to microbiological analysis by culture.

Each site was sample by serting paper points into the sulcus around the implant with peri-implantitis, in 14 implants, or by inflammatory soft tissue, in other 2 implants. Then the samples were grown in brain heart infusion broth.

Results: We found Gram-negative anaerobic bacteria, Fusobacterium nucleatum, in one sample, Prevotella oralis, in one sample, Peptostreptococcus, in one sample, and Stomatococcus, in 3 samples. In 9 samples were impossible to found one predominant bacteria. Antimicrobial susceptibility of isolates was 
determined. Antibacterial activity of amoxicillin-clavulanate was significantly higher than with other antimicrobials tested like amoxicillin or metronidazole.

Conclusions: This study found that microbiota associated with periimplantitis are Stomatococcus, Prevotella oralis, Peptostreptococcus and Fusobacterium nucleatum. Actinobacillus actinomycetemcomitans was not detected in any of the samples. Possible risk factors of peri-implantitis were hydroxyapatite, dental implants of $3,25 \mathrm{~mm}$ of diameter or distal position of implants in maxillary prosthetic superstructure.

\section{KEY-WORDS}

Dental implants, peri-implantitis/microbiology.

\section{CONCLUSIONES}

1. En nuestro estudio las bacterias asociadas a la periimplantitis han sido: Stomatococcus, Fusobacterium nucleatum, Prevotella oralis y Peptostreptococcus.

2. No se hallaron especímenes de Actinobacillus actinomycetemcomitans.

3. Todas las bacterias fueron muy sensibles a la asociación de amoxicilina y ácido clavulánico.

4. El control de placa bacteriana debe ser muy estricto en pacientes parcialmente edéntulos $\mathrm{y} / \mathrm{o}$ con antecedentes de enfermedad periodontal.

5. Los posibles factores de riesgo en nuestra serie han sido:

- Implantes recubiertos con hidroxiapatita.

- Implantes de 3,25 mm de diámetro.

- La sobrecarga oclusal en el implante situado en la posición más distal de las prótesis colocadas en los extremos libres edéntulos superiores.

- Antecedentes de enfermedad periodontal.

- Ser fumador de 15 cigarrillos al día como mínimo.

\section{BIBLIOGRAFÍA}

1. Nevins M, Jovanovic S. Localized bone reconstruction as an adjunct to dental implant placement. Curr Opin Periodontol 1997; 4: 109-18.

2. Albrektsson T, Isidor F. Consensus report of session IV. Proceedings of the first european workshop on periodontology. London: Quintessence, 1994.

3. Mombelli A. Etiology, diagnosis, and treatment considerations in peri-implantitis. Curr Opin Periodontol 1997: 4:127-36.

4. Jovanovic S. The management or peri-implant breakdown around functioning osseointegrated dental implants. J Peridontol 1993; 64: 1176-83.

5. Mombelli A, Van Oosten MAC, Schürch E, Lang NP. The microbiota associated with successful or failing ossteointegrated titanium implants. Oral Microbiol Inmunol $1987 ; 2: 145-51$.

6. Mombelli A, Maxer M, Gaberthüel T, Grunder U, Lang NP. The microbiota of osseointegrated implants in patients with a history of periodontal disease. J Clin Periodontol 1995: 22; 124-30.

7. Delgado Molina E, Sánchez Garcés MA, Rumeu Milá J, Berini Aytés L, Gay Escoda C. Enfermedad periimplantaria. Etiología, fisiopatología y diagnóstico. Revisión de la literatura. Arch Odontoestomatol 1999; 2: 53-67.

8. Tillmanns H, Hermann J, Tiffee J, Burgess A, Meffert R. Evaluation of three different dental implants in ligatureinduced peri-implantitis in the beagle dog. Part II. Histology and microbiology. Int J Oral Maxillofac Implants 1998; 13: 59-69.

9. Rosenberg ES, Torosian JP, Slots J. Microbial differences in two clinically types of failures of osseointegrated implants. Clin Oral Impl Res 1991; 2: 135-44.

10. Melloning JT, Griffiths G, Mathys E, Spitznagel J. Treatment of the failing implant: Case reports. Int J Periodont Rest Dent 1995; 15: 385-95.

11. Delgado E, Sánchez MA, Berini L, Gay Escoda C. Manejo clínico y tratamiento de la infección periimplantaria. Presentación de 6 casos. Av Periodoncia 1998; 10: 143-53.

12. Meffert RM. Treatment of the failing implant. CDAJ 1992; 20: 42-5.

13. Esposito M, Hirsch J, Lekholm U, Thomsen P. Differencial diagnosis and treatment strategies for biologic complications and failing oral implants: A review of the litera- 
ture. Int J Oral Maxillofac Implants 1999; 14: 473-90.

14. Lindhe J, Berglundh T, Lilenberg B, Marinello C. Experimental breakdown of periodontal tissues: a study in the beagle dog. Clin Oral Impl Res 1992, 3: 916.

15. Adell R, Eriksson B, Lekholm U, Brånemark PI, Jemt T. A long-term follow-up study of osseointegrated implants in the treatment of totally edentulous maxillary. Int Oral Maxillofac Implants 1990; 5: 347-59.

16. Hickey JS, O'Neal RB, Sheidt MJ, Strong SL, Turgeon D, Van Dyke TE. Microbiologic characterization of ligature-induced peri-implantitis in the microswine model. J Periodontol 1991; 62: 548-53.

17. Mombelli A, Buser D, Lang NP. Colonization of ossteointegrated implants in edentulous patients: early results. Oral Microbiol Inmunol 1988; 3:113-20.

18. Hultin M, Boström L, Gustafsson A. Neutrophil respose and microbiological findings around teeth and dental implants. J Periodontol 1998; 69: 1413- 8.

19. Leonhardt A, Berglundh T, Ericcson I, Dahlen G. Putative periodontal pathogens on titanium implants and teeth in experimental gingivitis and periodontitis in beagle dogs. Clin Oral Impl Res 1992; 3: 112-9.

20. Sbordone L, Barone A, Ramaglia L, Ciaglia RN, Iacono V. Antimicrobial suseptibility of periodontopathic bacteria associated with failing implants. J Periodontol 1995; 66: 69-74.

21. Oringer J, Palys MD, Iranmanesh A, Fiorellini JP, Haffajee AD, Socransky SS, Giannobile WV. C-Telopeptide pyridinoline cross-links (ICTP) and periodontal pathogens associated with endosseous oral implants. Clin Oral Impl Res 1998; 9: 365 -73.

22. Teanpaisan R, Douglas CWI, Eley AR, Walsh TF. Clonality of Porphyromonas gingivalis, Prevotella intermedia and Prevotella negrescens isolated from periodontally diseased and healthy sites. J Periodont Res 1996; 31: 423-32.

23. Leonhardt A, Adolfsson B, Lekholm U, Wikstrom M. A longitudinal microbiological study on osseointegrated titanium implants in partially edentulous patients. Clin Oral Impl Res 1993; $4: 113-20$.

24. Sneath PH. Bergey's manual of systematic bacteriology. Vol 2. Baltimore: Williams \&Wilkins, 1986: 1008-11, 1083-93.

25. Gay Escoda C, Berini Aytés L. Infección odontógena. Madrid: Ergon, 1997: 1-12.
26. Davis BD, Dulbecco R, Eisen HN, Ginsberg HS. Tratado de microbiología. $4^{a}$ ed. Barcelona: Masson, 1990: 56771, 697-706.

27. Takemoto T, Hino T, Yoshida M, Nakanishi K, Shirakawa M, Okamoto H. Characteristics of multimodal co-aggregation between Fusobacterium nucleatum and streptococci. J Periodont Res 1995; 30: 252-7.

28. Eke PI, Braswell L, Fritz M. Succession of putative periimplant pathogens after root-form and plate-form implant placement in partially dentate adult monkeys. J Periodont Res 1995; 30: 88-96.

29. Quirynen M, Listgarten M. The distribution of bacterial morphotypes around teeth and titanium implants ad modum Brånemark. Clin Oral Impl Res 1990; 1: 8-12.

30. Aspe P, Ellen RP, Overall CM, Zarb GA. Microbiota and crevicular fluid collagenase activity in the osseointegrated dental implants sulcus: a comparison of sites in edentulous and partially edentulous patients. J Periodontal Res 1989; 24: 96-105.

31. George K, Zafiropoulos G-GK, Murat Y, Hubertus S, Nisengard RJ. Clinical and microbiological status of osseointegrated implants. J Periodontal 1994; 65: 76670 .

32. Talarico G, Neiders M, Comeau R, Cohen R. Phenotypic characterization of mononuclear cells from gingiva associated with periodontitis and peri-implantitis. J Oral Implantol 1997; 1: 5-11.

33. Ericsson I, Persson LG, Berglundh T, Edlund T, Lindhe J. The effect of antimicrobial therapy on peri-implantitis lesions. An experimental study in the dog. Clin Oral Impl Res 1996; 7: 320-8.

34. Persson LG, Araújo MG, Berglundh T, Gröndahl K, Lindhe J. Resolution of peri-implantitis following treatment. Clin Oral Impl Res 1999; 10: 195-203.

35. Van Winkelhoff AJ, Rodenburg JP, Groerené RJ, Abbas F, Winkel EG, de Graaf J. Metronidazole plus amoxycillin in the treatment of Actinobacillus actinomycetemcomitans associated periodontitis. J Clin Periodontol 1989; 16: 128-31.

36. Saadoun AP, Le Gall M, Krichech M. Microbial infections and oclusal overload: Causes of failure in osseointegrated implants. Pract Periodont Aesthet Dent 1993; 5: 11-20.

37. Rangert B, Eng M, Sullivan R, Jemt T. Load factor control for implants in the posterior partially edentulous segment. Int J Oral Maxillofac Implants 1997; 12: 360-70.

38. Quiryinen M, Naert I, Van Steenberghe D. Fixure desing 
and overload influence marginal bone loss and fixure success in the Brånemark system. Clin Oral Impl Res 1992; 3: 104-11.

39. Lindquist LW, Rockler B, Carlison GE. Bone resorption around fixure in edentulous patients treated with mandibular fixed tissue-integrated prosthesis. J Prosthet Dent 1988; 59: 59-63.

40. Jaffin RA, Berman CL. The excessive loss of Branemark fixtures in type IV bone: A 5-years analysis. J Periodontol 1991; 62: 2-4.

41. Hutton JE, Heath MR, Chai JY, Harnet J, Jemt T, Johns B et al. Factors related to success and failure rates at 3-years follow-up in multicenter study of overdentures supported by Brånemark implants. Int J Oral Maxillofac Implants 1995; 10: 33-42.

42. Ogiso M, Tabata T, Kuo PT, Borgese D. A histological comparison of the functional loading capacity of an occluded dense apatite implant and the natural dentition. J Prosthet Dent 1994; 71: 581-8.

43. Rams TE, Roberts TW, Tatum H, Keyes PH. The subgingival microbial flora associated with man dental implants. J Prosthet Dent 1984; 51: 529-34.

44. Van Steenbergen TJM, Bosch-Tijhof CJ, Petit MDA, Van de Velden D. Intrafamilial transmission and distribution of Prevotella intermedia and Prevotella nigrescens. J Periodont Res 1997; 32: 345-50.

45. Slaughter T, Babbush C, Langer B, Buser D, Holmes R. Solutions for specific bone situations. Int J Oral Maxillofac Implants 1994; 9: 19-29.

46. Ichikawa $\mathrm{T}$, Hirota $\mathrm{K}$, Miyake $\mathrm{Y}$, Matsumoto $\mathrm{N}$. In vitro adherence of Streptococcus constellatus to dense hydroxyapatite and titanium. J Oral Rehabil 1998; 25: 125-7.
47. Wu-Yuan C, Eganhouse $\mathrm{K}$, Keller J, Walters K. Oral bacterial attachment to titanium surfaces: A scanning electron microscopy study. J Oral Implant 1995; 3: 207-13.

48. Lekholm U, Zarb GA. Selección y preparación del paciente. En: Brånemark PI, Zarb GA, Albrektsson T. Prótesis tejido-integradas. La osteointegración en la odontología clínica. Berlin: Quintessence, 1987:199209.

49. Jovanovic S. Diagnosis and treatment of peri-implant disease. Currr Opin Periodontol 1994; 194-204.

50. Meffert RM. Do implant surfaces make a difference?. Curr Opin Periodontol 1997; 4: 104-8.

51. Tarnow DP. Dental implants in periodontal care. Curr Opin Periodontol 1993; 157-62.

52. Salcetti JM, Moriarty JD, Cooper LF, Smith FW, Collins JG, Socransky SS, Offenbacher S. The cinical microbial and host response characteristics of the failing implant. Int J Maxillofac Impl 1997; 12: 32-42.

53. Salvi GE, Williams RC, Offenbacher S. Nonesteroidal anti-inflamatory drugs as adjuncts in the management of periodontal diseases and peri-implantitis. Curr Opin Periodontol 1997; 4: 51-8.

54. Denninson DK, Huerzeler MB, Quinones C, Caffesse RG. Contamined implant surface: An in vitro comparison of implant surface coating and treatment modalities for decontamination. J Periodontol 1994; 65: 942-8.

55. Bain CA. Smoking and implant failure-benefits of a smoking cessation protocol. Int J Oral Maxillofac Implants 1996; 11: 756-9.

56. Haas R, Haimbock W, Mailath G, Watzek G. The relationship of smoking on peri-implant tissue: a retrospective study. J Prosthet Dent 1996; 76: 592-6. 